\title{
The Effect of Tramadol on Decreasing Postoperative Shivering
}

\author{
${ }^{1}$ Ghazi S. Aldehayat, ${ }^{2}$ Maisa K. Abu Kamar and ${ }^{1}$ Adnan A. Abu Kamar \\ ${ }^{1}$ King Hussein Medical Center, Amman, Jordan \\ ${ }^{2}$ Department of Biopharmaceutics and Clinical Pharmacy, \\ Faculty of Pharmacy, University of Jordan, Amman, Jordan
}

\begin{abstract}
Shivering is a well-recognized postoperative complication. The objective of the current study is to evaluate the role of tramadol in decreasing post anesthetic shivering. About 80 patients ASA I and II adult patients, aged between 18-70 years, undergoing general anesthesia were enrolled in this prospective study. They were divided into 2 groups, group $1(\mathrm{n}=40)$ received $1 \mathrm{mg} / \mathrm{kg}$ of tramadol towards the end of the operation while group $2(n=40)$ received normal saline. Shivering was evaluated during the postoperative period. No difference among patients regarding patient characteristics. In group 1, postoperative shivering was effectively prevented in $36(90 \%)$ and $38(95 \%)$ patient at 15 and 30 min, respectively and none of patients shivered at grade $=3$ and grade $=2$. However, in group 2, the incidence of shivering was 50 and $57.5 \%$ at 15 min and 30 , respectively, the difference between 2 groups was statistically significant $(\mathrm{p}<0.05)$. Tramadol, when given at the end of the operation can decrease the incidence of shivering.
\end{abstract}

Key words: Tramadol, shivering, postoperative, regarding, characteristics, anesthesia

\section{INTRODUCTION}

Postanesthetic shivering is one of the most common postoperative complications. It involves involuntary movements of one or more groups of muscles and with reported incidence as high as 65\% (Hemmati et al., 2014). Shivering is one of the important complications of intraoperative inadvertent hypothermia it is due to abnormal muscle activity result from intraoperative hypothermia or secondary chills due to activation of inflammatory response and cytokine release also thermoregulatory control can be impaired during general anesthesia.

Shivering from any cause results in sympathetic stimulation and increases myocardial oxygen consumption which may lead to myocardial ischemia (De-Witte and Sessler, 2002) also shivering causes considerable discomfort in awake postoperative patients.

Postoperative shivering is typically, treated with meperidine intravenous (IV). A reasonable alternative are tramadol an alpha $_{2}$ agonist such as clonidine, dexmedetomidine (Choi et al., 2017). Tramadol Hydrochloride $(\mathrm{HCl})$ is a synthetic opioid that acts through the inhibiting the re-uptake of 5Hydroxy Tryptamine (5-HT, serotonin), norepinephrine and dopamine as well it modulates the activity of the nucleus median raphe in the medulla acting centrally on the $\mathrm{m}$ opioid receptor. The current study has been conducted to evaluate the role of tramadol in decreasing the incidence of postoperative shivering.

\section{MATERIALS AND METHODS}

About 80 patients undergoing various moderate surgical procedures under general anesthesia were enrolled in this study after the approval of local ethical committee. All patients were with physical status ASA 1 and 2 and were satisfied with the general anesthesia in King Hussein Medical Center Hospital, Amman, Jordan from 2018-2019. Exclusion criteria included patients with a history of history of coronary artery disease or cardiorespiratory or neuromuscular pathology or who require administration of blood or blood products during procedure. The patients were randomly allocated into 2 groups. Group $1(\mathrm{n}=40)$ received $1 \mathrm{mg} / \mathrm{kg}$ tramadol and group $2(n=40)$ received normal saline toward the end of surgery.

The Anesthetic technique was standardized for all included patients. Patients were prepared by $8 \mathrm{~h}$ preoperative fasting. After the patients were brought into the operating room, the monitors were attached and baseline values for Pulse Rate (PR), Mean Arterial Pressure (MAP) and $\mathrm{O}_{2}$ saturation were recorded. Following that induction of anesthesia was carried out with sleeping by propofol $2 \mathrm{mg} / \mathrm{kg}$. Endotracheal intubation was facilitated using rocuronium $1 \mathrm{mg} / \mathrm{kg}$. The maintenance of anesthesia was achieved by $1.5-2 \%$ sevoflurane with $50 \%$ oxygen in air. Remifentanil infusion has been used to maintain intraoperative analgesia. Toward the end of the operation the patients in group one received tramadol $1 \mathrm{mg} / \mathrm{kg}$ while patents in

Corresponding Author: Maisa K. Abu Kamar, Department of Biopharmaceutics and Clinical Pharmacy, Faculty of Pharmacy, University of Jordan, Amman, Jordan 
Res. J. Med. Sci., 13 (6): 115-117, 2019

Table 1: Grade of shivering

\begin{tabular}{ll}
\hline Grade & Clinical sign \\
\hline 0 & No shivering \\
1 & Mild fasciculation of face or neck \\
2 & Visible tremor involving more than one muscle group \\
3 & Gross muscular activity involving entire body \\
\hline
\end{tabular}

group 2 received normal saline. Temperature probe was inserted into the esophagus. The temperature of the operating room was maintained between $22-24^{\circ} \mathrm{C}$.

Patients were observed in the recovery room for $30 \mathrm{~min}$. Degree of post anesthetic shivering was noted every fifteen minutes by an observer who was unaware of the nature of the surgery. Shivering was graded using a scale similar to that validated by Badjatia et al. (2008) as shown in Table 1 . The collected data were then entered into the SPSS Statistics V24.0 Software program and analyzed via. Chi-Squared tests and ANOVA tests.

\section{RESULTS AND DISCUSSION}

Demographic profile and preoperative vital parameters were comparable in both groups (p-value $>0.05$ ) (Table 2 and 3 ). In group 1 , postoperative shivering was effectively prevented in $36(90 \%)$ and 38 (95\%) patient at 15 and 30 min, respectively and none of patients shivered at grade $=3$ and grade $=2$. However, in group 2, the incidence of shivering was 50 and $57.5 \%$ at $15 \mathrm{~min}$ and 30, respectively. The temperature of the patients was maintained $>36^{\circ} \mathrm{C}$ all through the procedure (Table 4 and 5). There is significant statistical difference between the 2 groups in the incidence of shivering. In the other words, incidence of shivering in the normal saline group was highly significant at the different time periods ( $\mathrm{p}<0.05$ at both time periods).

Postoperative shivering is a frequent complication of anaesthesia it has been reported to range from $20-70 \%$ in general anaesthesia (Eberhart et al., 2005). Shivering is believed to increase oxygen consumption and increase the risk of hypoxemia it might also increase postoperative complications (Alfonsi, 2003).

A number of factors including age, duration of surgery, gender and temperature of the operating room are risk factors for shivering (Witte and Sessler, 2002) so, the temperature of operation room was maintained at $24^{\circ} \mathrm{C}$. Volatile agents have been found to be associated with more incidence of postoperative shivering than intravenous anesthetic agents (Horn et al., 1998). This may explain the high incidence of shivering that has been found in our study as we have used sevoflurane as anesthetic agent.

Various drugs have been used to treat or prevent postoperative shivering. Meperidine, tramadol, ketamine, physostigmine (Choi et al., 2017; Alfonsi, 2003) have been effectively used in prevention and treatment of
Table 2: Demographic and intraoperative variables of the subjects (mean and ranges)

\begin{tabular}{lll}
\hline Variables & Group 1 & Group 2 \\
\hline Age (years) & $39(18-70)$ & $40(19-68)$ \\
Sex (male: female) & $22 / 18$ & $20 / 20$ \\
Weight (kg) & $70(49-92)$ & $69(51-95)$ \\
Duration surgery (min) & $140(112-172)$ & $150(108-168)$ \\
\hline
\end{tabular}

Table 3: Vital signs values across procedure (mean \pm standard deviation)

\begin{tabular}{lrl}
\hline Variables & Group 1 & Group 2 \\
\hline HR (beats/min) & $80 \pm 5.2$ & $83 \pm 4.8$ \\
SBP (mm Hg) & $110 \pm 2.03$ & $115 \pm 2$ \\
DBP (mm Hg) & $75 \pm 2.01$ & $77 \pm 2.8$ \\
$\mathrm{SPO}_{2}(\%)$ & $98 \pm 1.01$ & $98 \pm 1.5$ \\
\hline
\end{tabular}

Table 4: Incidence and severity of postoperative shivering at $15 \mathrm{~min}$ after tramadol and normal saline administration

\begin{tabular}{lcc}
\hline Grade of shivering & Group $1(\mathrm{n}=40)$ & Group 2 $(\mathrm{n}=40)$ \\
\hline 0 & $36(90 \%)$ & $20(50 \%)$ \\
1 & $4(10 \%)$ & $15(37.5 \%)$ \\
2 & $0(0 \%)$ & $3(7.5 \%)$ \\
3 & $0(0 \%)$ & $2(5 \%)$ \\
\hline
\end{tabular}

Table 5: Incidence and severity of postoperative shivering at $30 \mathrm{~min}$ after tramadol and normal saline administration

\begin{tabular}{lcc}
\hline Grade of shivering & Group 1 $(\mathrm{n}=40)$ & Group 2 (n=40) \\
\hline 0 & $38(95 \%)$ & $23(57.5 \%)$ \\
1 & $2(5 \%)$ & $15(37.5 \%)$ \\
2 & $0(0 \%)$ & $2(5 \%)$ \\
3 & $0(0 \%)$ & $0(0 \%)$ \\
\hline
\end{tabular}

postoperative shivering. In our study there was no significant statistical difference between the tramadol and control groups in terms of demographic data and vital parameters (heart rate, systolic blood pressure, diastolic blood pressure and $\left.\mathrm{PO}_{2}(\mathrm{p}>0.05)\right)$. However, this study found significant statistical difference between tramadol and control groups in terms of shivering incidence.

Our results are inconsistent with results of many other studies conducted in this area. For instance, there was a randomized clinical trial conducted by Mathews et al. (2002) that found tramadol to be superior to pethidine without any incidence of severe side-effects in preventing postanesthetic shivering (Mathews et al., 2002) another study reported the incidences of shivering in both tramadol groups were significantly lower than the groups that did not receive tramadol (Nakagawa et al., 2017). While a study conducted by Venkatraman et al. (2018) found that dexmedetomidine is more effective than tramadol and clonidine in the treatment of shivering because of faster onset, lesser recurrence rate and better sedation (Venkatraman et al., 2018). Tramadol, m opioid receptor agonist which has the best combination of anti-shivering and analgesic efficacy without excessive sedation and significant side-effects.

In our study tramadol hydrochloride $1 \mathrm{mg} / \mathrm{kg}$ IV was given at the end of procedure and postoperative shivering was effectively prevented in more than $90 \%$ of patients (Table 4 and 5). Shivering of grades 2-3 was not observed in any of the patients received tramadol. 


\section{CONCLUSION}

In conclusion, tramadol hydrochloride is effective and safe in preventing postoperative shivering following open operations in a dose of $1 \mathrm{mg} / \mathrm{kg}$.

\section{REFERENCES}

Alfonsi, P., 2003. Postanaesthetic shivering. Epidemiology, pathophysiology and approaches to prevention and management. Minerva Anestesiologica, 69: 438-442.

Badjatia, N., E. Strongilis, E. Gordon, M. Prescutti and L. Fernandez et al., 2008. Metabolic impact of shivering during therapeutic temperature modulation: The bedside shivering assessment scale. Stroke, 39: 3242-3247.

Choi, K.E., B. Park, A.M. Moheet, A. Rosen, S. Lahiri and A. Rosengart, 2017. Systematic quality assessment of published antishivering protocols. Anesth. Analg., 124: 1539-1546.

De-Witte, J. and D.I. Sessler, 2002. Perioperative shivering: Physiology and pharmacology. Anesthesiology, 96: 467-484.

Eberhart, L.H., F. Doderlein, G. Eisenhardt, P. Kranke and D.I. Sessler et al., 2005. Independent risk factors for postoperative shivering. Anesth. Analg., 101: 1849-1857.
Hemmati, K., E. Derikvand, A. Delpisheh and M. Safari, 2014. Comparison between effects of different doses of pethidine and fentanyl on shivering after spinal anesthesia in orthopedic patients referred to Imam Khomeini Hospital (RH) of Ilam. Anesthesiology Pain, 5: 40-48.

Horn, E.P., D.I. Sessler, T. Standl, F. Schroeder, H.J. Bartz, J.C. Beyer and J.S.A. Esch, 1998. Non-thermoregulatory shivering in patients recovering from isoflurane or desflurane anesthesia. Anesthesiology, 89: 878-886.

Mathews, S., A. Al Mulla, P.K. Varghese, K. Radim and S. Mumtaz, 2002. Postanaesthetic shivering-a new look at tramadol. Anaesthesia, 57: 387-403.

Nakagawa, T., M. Hashimoto, Y. Hashimoto, K. Shirozu and S. Hoka, 2017. The effects of tramadol on postoperative shivering after sevoflurane and remifentanil anesthesia. BMC. Anesthesiology, Vol. 17, No. 1. 10.1186/s12871-016-0295-x

Venkatraman, R., K. Karthik, A. Pushparani and A. Mahalakshmi, 2018. A prospective, randomized, double-blinded control study on comparison of tramadol, clonidine and dexmedetomidine for post spinal anesthesia shivering. Rev. Bras. Anestesiologia, 68: 42-48.

Witte, J.D. and D.I. Sessler, 2002. Perioperative shivering physiology and pharmacology. Anesthesiology, 96: 467-484. 